\title{
Effect of marital status on death rates. Part 1: High accuracy exploration of the Farr-Bertillon effect.
}

\section{Peter Richmond ${ }^{1}$ and Bertrand M. Roehner ${ }^{2}$}

\begin{abstract}
The Farr-Bertillon law says that for all age-groups the death rate of married people is lower than the death rate of people who are not married (i.e. single, widowed or divorced). Although this law has been known for over 150 years, it has never been established with great accuracy. This even let some authors argue that it was a statistical artifact. It is true that the data must be selected and analyzed with great care, especially for age groups of small size such as widowers under 25 .

The observations reported in this paper were selected and designed in the same way as experiments in physics, that is to say with the objective of minimizing the error bars for all age-groups. It will be seen that data appropriate for mid-age groups may be unsuitable for young age groups and vice versa.

The investigation led to the following results. (1) The FB effect is basically the same for men and women, except that on average it is about $20 \%$ stronger for men. (2) There is a marked difference between single or divorced persons on the one hand, for whom the effect is largest around the age of 45, and widowed persons on the other hand, for whom the effect is largest around the age of 25. (3) When different causes of death are distinguished, the effect is largest for suicide and smallest for cancer. (4) For young widowers the death rates are up to 10 times higher than for married persons of same age. This extreme form of the FB effect will be referred to as the "young widower effect".
\end{abstract}

A possible connection between the FB effect and Martin Raff's "Stay alive" effect for cells in an organism is discussed in the last section.

\section{Version of 19 August 2015. Comments are welcome.}

Key-words: death rate, marital status, widowhood, young widowers, apoptosis.

1: School of Physics, Trinity College Dublin, Ireland. Email: peter_richmond@ymail.com

2: Institute for Theoretical and High Energy Physics (LPTHE), University Pierre and Marie Curie, Paris, France. Email: roehner@1pthe.jussieu.fr 


\section{Introduction}

Let us first define several terms which will be used throughout this article.

- The marital status of a person refers to one of the following situations: single, married, widowed, divorced. Needless to say, "single" means that the person has never been married for otherwise he (or she) would be widowed or divorced. These groups will be designated by the letters $s, m, w, d$ respectively. The case of people who are married but separated or not married but cohabiting will also be considered later on albeit fairly shortly.

- For each of these groups of persons one can define a death rate in the standard way, that is to say by dividing the number of persons who die annually by the size of the group. In addition to the marital status distinction, one can order people by age group. For instance, $d_{m}(15: 24)$ will be the death rate of married persons who are between 15 and 24 year old.

- Finally, we introduce the notion of death rate ratio which is the death rate of a given group divided by the death rate of married persons of same age. For instance, the death rate ratio of widowed persons in the age group $15: 24$ will be:

Death rate ratio of widowed persons: $\quad r_{w}(15: 24)=d_{w}(15: 24) / d_{m}(15: 24)$

The expression death rate ratio distribution of widowed persons will refer to the curve of $r_{w}$ as a function of age. Sometimes, death rate ratio distributions will also be named Farr-Bertillon distributions.

\section{The Farr-Bertillon law}

In the social sciences there are very few laws which are valid at any time and in any country. The Farr-Bertillon law 1 which states that for all age-groups married persons have a lower death rate than unmarried persons is one of them. More precisely, in all cases for which reliable data are available this law holds with error bars which are not broader than $\pm 10 \%$.

At first sight, our assertion that there are few laws of this kind may seem surprising. For instance, is it not true that the frequency distribution of high incomes follows a Pareto law? Compared with the Farr-Bertillon law there are two major differences, however.

- The Pareto law contains a free parameter, namely the exponent of the power law. The Farr-Bertillon effect contains no free parameter.

\footnotetext{
${ }^{1}$ So far, in the literature the FB law was variously referred to as the "marriage effect" , the "widower effect" or the "bereavement effect". Adding to the confusion, some of these expressions were meant to describe special facets; for instance the term "bereavement effect" focuses on short-term rather than permanent effects. Here, as is standard in physics, this law will be designated by the name of its discoverers. We hope that following this usage will clarify its significance.
} 
- The Pareto law describes a frequency distribution whereas the Farr-Bertillon law is a relationship between two "physical" variables. In short, the Pareto law is of the same kind as the Maxwell-Boltzmann law which gives the velocity distribution of the molecules of a gas whereas the Farr-Bertillon law is similar (for instance) to Einstein's law which gives the relationship between specific heat and temperature. Needless to say, a relationship between physical variables tells us more about the system than a probability distribution? 2 .

The Farr-Bertillon law consists in the fact that married people have lower death rates than non-married people, either never married, widowed or divorced people. It is named after William Farr (1807-1883) and Louis-Adolphe Bertillon (1821-1883). In 1859 Farr observed the effect on French data. Both Farr and Bertillon were among the main founders of medical demography. Bertillon's strong focus on comparative international investigations led him to recognize the existence of this effect in a broad range of countries (Bertillon 1872). As a matter of fact, in the one and a half century since its discovery, the Farr-Bertillon effect has been observed in all countries for which reliable data are available.

\section{Measurement issues}

Why did we emphasize that the data must be reliable?

Even today in industrialized countries it remains a real challenge to produce the data which are needed to observe this law. It can even be said that present-day data are probably less accurate than those of 50 or 100 years ago for, as will be seen later, present-day statistics rely more and more on surveys based on population samples.

Why is it a difficult task to measure the death rate of widowed persons and particularly of young widowers? As all death rates, the death rate $d(G)$ of widowers in age-group $G$ is defined as a ratio:

$$
d(G)=\frac{\text { Number of widowers who died during the year: } D(G)}{\text { Population of widowers at beginning of year in given age group: } P(G)}
$$

The numerator is fairly easy to measure because in all countries age and marital status are two characteristics recorded on death certificates. In contrast, it is difficult to get reliable estimates for $P(G)$. There are (at least) 4 difficulties in measuring $d(G)$ for young widowers.

(1) In the age groups under 30 there are few widowers. For instance in 1980 in the United States the 15-19 age group had only 6,448 widows and 2,081 widowers. In

\footnotetext{
${ }^{2}$ The MB distribution for the speed of molecules is a consequence of the fact that each velocity component follows a centered Gaussian distribution. Because of the central limit theorem, Gaussian distributions are very common in the natural sciences which means that the exponential shape of the MB distribution only tells us that it belongs to this broad class rather than to the power law class. Actually, all significant physical information (about molecules masses and temperature) is contained in the width of the MB distribution.

${ }^{3}$ There are less widowers than widows because at the same age there are less married men than married women.
} 
the 15-24 age group there were 31,100 widows and 8,050 widowers. In any census the task of identifying and counting accurately such small populations is not easy. Naturally, if instead of real censuses one relies on sample surveys, the task becomes even more difficult or altogether impossible if the samples are too small.

(2) Even though in a general way it is easier to measure death numbers than to count living persons, the fact that there are very few deaths of young widowers creates huge statistical fluctuations. Thus, in the United States in 1980 for the agegroup 15-24 there were only 38 deaths of widows and 29 deaths of widowers. In the following age group 25-34 the numbers were about ten times larger, 323 and 219 respectively, but these are still small numbers. As a matter of fact, the deaths of widowed persons become "substantial" only over 65 years of age. Thus, in the age group 65-74 there were 35,630 deaths of widowers.

(3) $P(G)$ must be measured through a census but the problem with censuses is that they are based on the answers provided by the respondents. Even in countries such as the United States where censuses have been organized with much care, the enumerator relies entirely on the answers provided by the head of the household 4 . Yet, it is well known that the answers provided by the respondents are not always accurate. For instance, even such basic variables as age or the number of years spent in widowhood are not well remembered especially by elderly people. It appears that often such variables are rounded up to the nearest multiples of 10 or 5 . That is why most census forms ask both the age and the year of birth. Moreover, the answers may be affected by other forms of bias. Thus, people may prefer to say that they are widowed rather than separated or divorced.

(4) In the interval between census years, most national statistical institutes carry out surveys based on population samples. The quality of such surveys greatly depends upon how well the samples are selected. In the United States, the annual "Current Population Reports" (CPR) are based on samples of some 60, 000 persons, that is to say one per 20,000. Thus, even if the sample was selected properly, there will be substantial sampling errors. For a population of the order of 100,000 the sampling error of the CPR of 1960 was 37,000 that is to say nearly 40\%; for a population of one million the standard error was still 12\% (Accuracy 1960, p. 6, Table D). As a result, good measurements of $d(G)$ for young widowers can be obtained only in census years. A more detailed analysis is given below in Table $3 \mathrm{~b}$.

\section{Farr-Bertillon effect in the 19th century}

In this subsection and in the next we show some of the results due to Louis-Alphonse Bertillon. As already observed, contrary to William Farr, Bertillon was a compara-

\footnotetext{
${ }^{4}$ In fact, after 1990 the census forms were mailed to the persons; visits by enumerators were limited to a few households.
} 
tivist. After having identified this effect in France, his main concern was to see if it was also present in other countries for which data were available.
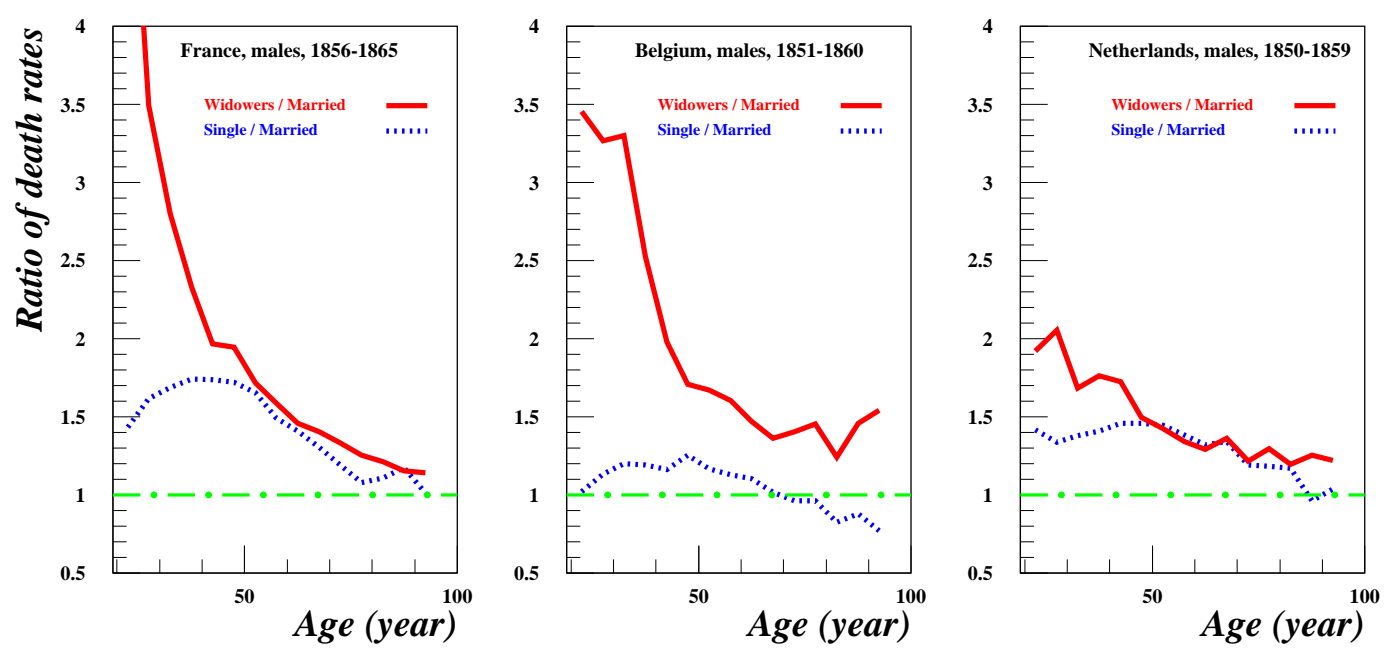

Fig. 1a,b,c: Death rate ratio according to marital status. The data points correspond to 16 age groups ranging from 20-25 to 95-100. It can be seen that the ratio widowers/married differs from the ratio single/married both in shape and in magnitude. Incidentally, Bertillon was suspecting a possible statistical bias for the death rates of single persons in Belgium because they are two thirds the size of those in France and the Netherlands. In this graph as in the rest of the paper "single" means "never married". Source: Bertillon (1872); the graph for France appears also in Bertillon (1879, p. 781)

\section{Bonds between parents and children}

If the bond between husband and wife plays a role in the FB effect it seems plausible to expect the ties between parents and their children to have a similar effect. This conjecture is confirmed by the data in Table 1 .

\section{Toward accurate observations of the Farr-Bertillon law}

\section{Mid-age groups versus young age groups}

In the previous section we emphasized the fact that the Farr-Bertillon effect holds with a level of precision akin to what one is used to in the natural sciences. However, in order to reduce the error bars as much as possible an appropriate methodology must be be used. In this respect age-groups over 35 and age-groups under 35 will require different techniques.

- To estimate the sizes of the age groups over 35 one does not necessarily need to use censuses. Estimates from surveys based on population samples may be sufficient at least if the samples are "not too small". This will allow observations over time intervals containing a substantial number, $n$, of inter-census years. For the averages computed over such time intervals, the error bars will be reduced by the standard 
Table 1: Effect of husband-wife ties and parents-children ties on suicide rates.

\begin{tabular}{llcccc}
\hline \hline Situation & M & F & M & F \\
\cline { 2 - 6 } & Married with children & 20 & 4.5 & 1 & 1 \\
Married without children & 47 & 16 & 2.4 & 3.6 \\
Widowed with children & 52 & 10 & 2.6 & 2.2 \\
Widowed without children & 100 & 23 & 5.0 & 5.1 \\
\hline
\end{tabular}

Notes: The table gives average suicide rates (per 100,000 people) in France over the 8-year time interval between 1861 and 1868. "M" means male and " $F$ " female. The two columns on the righthand size repeat the same data with a normalization based on the situation "married with children". If one accepts the explanation introduced by Emile Durkheim (1897) that it is the severance (or lack) of bonds and especially of family bonds which is the main factor in the phenomenon of suicide, then these data allow us to compare the respective strengths of the bonds between husband and wife on the one hand and between parents and children on the other hand. The fact that the suicide rate is almost the same for married persons without children as for widowed persons with children suggests that the parents-children and husband-wife bonds are of same strength.

Source: Bertillon (1879, p. 474)

$1 / \sqrt{n}$ factor.

- On the contrary, in the investigation of the young widower effect one needs to focus on age groups under 35 and, as already mentioned, this requires to rely on population data from decennial censuses. To some extent, the procedure based on census data is also needed for elderly age groups over 75 because of their small size.

In short, there will be two phases in our investigation. In a first phase we will focus on the central part of the age interval (30-60) and use as many years as possible to get the smallest error bars.

In the second phase, we will use accurate population data available for only a few years. This will give the death ratio for young age-groups. Though this procedure will of course also provide results for central age-groups, they will be less precise than those computed in the first phase.

\section{Methodological options}

In the present paper we perform repeated observations. At first sight one may think that they should be aggregated together. However, as these observations are not performed under identical conditions (see table 1) lumping them together would lead to unpredictable and uncontrollable results. As explained below, this is a widespread difficulty in the social sciences.

As a matter of fact, nothing is more depressing than to read sociological review papers. Why?

Most often, the authors of such papers report conflicting results obtained by different researchers but without describing the conditions under which the observations were 
made and how the data were analyzed. Inevitably, this makes readers fairly uncomfortable. One gets the feeling of being confronted with a soft, multiform, shapeless and labile world about which no clear, univocal statement can ever be made. Needless to say, if true, such a view would condemn any scientific investigation from the outset because reproducibility is a crucial requirement in any science.

Let us illustrate this point through an example. A paper by Koposawa et al. (1995) found that no additional risk of suicide is significantly associated with the marital status of widowed or never-married persons. Such a conclusion is at variance with the results reported consistently by numerous former and subsequent studies including the present one. If presented without specific explanations about its methodology, this study would give the impression that even the most unlikely claim can be made and sustained. A closer look reveals that, in contrast with most other investigations, this one does not rely on aggregated data but on a multivariate analysis of individual data. The sample contains only 216 suicide cases. As such a small sample implies broad confidence intervals it is hardly surprising that the study could not find any significant connection between marital status and suicide rates. This does not mean that the connection does not exist but rather that the data used in this study were dominated by background noise.

Only observations of same nature and quality can possibly be lumped together. Thus, in the observations listed in Table 2 it would be possible to lump together the observations 3 and 4. However, from 1940 to 2000 they would span a time interval of 60 years during which important population changes took place in the United States. By keeping these observations separate one can control whether or not there was a possible shift.

Before carrying out the program outlined in Table 2, some preliminary tests are required. In the previous discussion we said that population estimates based on surveys may be acceptable provided that the samples are "not too small". Obviously, one needs to clarify what is meant by this expression. This will be done in the next section.

\section{Sampling errors for population estimates}

The expression "sampling errors" corresponds to measurement errors due to purely random fluctuations. However, we will see that for some sample estimates there are also non-sampling errors which refer to more or less systematic biases. As an example, one can mention the response rate. Nowadays, once the sample has been selected the forms are mailed to the respondents. Not all of them will reply, however. In the United States, response rates usually range between $80 \%$ and $95 \%$. The per- 
Table 2: Summary of the observations of the Farr-Bertillon effect

\begin{tabular}{llllllll}
\hline \hline Fig. & Country & Period & $\begin{array}{l}\text { Population } \\
\text { estimates }\end{array}$ & $\begin{array}{l}\text { Error } \\
\text { bars }\end{array}$ & $\begin{array}{l}\text { Shape } \\
\text { of w/m }\end{array}$ & $\begin{array}{l}\text { Quality } \\
\text { stars }\end{array}$ \\
\hline 1a & France & $1856-1865$ & $?$ & no & $>$ & $*$ \\
1b & Belgium & $1851-1860$ & $?$ & no & $>$ & $*$ \\
1c & Netherlands & $1850-1859$ & $?$ & no & $<$ & $*$ \\
2a,b,c & USA & $1996-2010$ & $?$ & yes & $?$ & $* *$ \\
3a,b,c & USA & $1940,1950,1960$ & census & yes & $<$ & $* * *$ \\
4 & USA & $1980,1990,2000$ & census & yes & $<$ & $* * *$ \\
5 & USA & $2005-2010$ & ACS & yes & $<$ & $* * *$ \\
6 & USA & 1980,1990 & census & yes & $><$ & $* *$ \\
$7 \mathrm{a}$ & France & $1968-1993$ & $?$ & yes & & $* *$ \\
$7 \mathrm{~b}$ & France & $1981-1993$ & $?$ & no & $>$ & $*$ \\
\hline
\end{tabular}

Notes: The column "Population estimates" indicates how the populations by age and marital status have been estimated. ACS means "American Community Survey". An interrogation mark in this column means that the technical notes of the publication failed to explain how the estimates were computed. The column "Error bar" indicates if it was possible to estimate the standard deviation of the death rate ratios. The column "Shape of $w / \mathrm{m}$ " indicates whether the curve for the death rate ratio of widowers is steadily decreasing $(>)$ or shows a maximum for the second youngest age group $(<)$. It is the last case which prevails in the observations of highest quality. The column "Quality stars" gives an estimated quality index for each observation: three stars means highest quality.

It can be noted that similar death statistics by marital status and age are also available for England (see Mortality Statistics, review of the Registrar General, National Center for Health Statistics 1970, Registrar General 1971) and Germany (see Statistisches Jahrbuch 1978).

sons who do not respond most likely are "unstable" households who move frequently and for that reason may not have received the form and also elderly persons who are in hospital or nursing homes.

Table 3a describes US statistical sources. Their accuracy will then be ascertained through a number of tests performed in Table $3 b$.

\section{Discussion of computational methods for estimating populations}

Before we close this section about population estimates an additional observation is in order concerning computational methods.

At first sight it might seem that in the intervals between census years it is easy to compute the sizes of age-groups. Indeed, based on the numbers of deaths, marriages, divorces in each age-group, one should be able to predict the sizes of relevant agegroups. Such a procedure which would permit to follow each age group year after year until the next census may work in some countries, but in the United States it does not. There are three main obstacles.

(1) One does not know the flows of illegal immigrants. Although this difficulty exists in all countries it is more or less serious depending on the magnitude of illegal 
Table 3a: Statistical sources for US population by marital status and age

\begin{tabular}{lll}
\hline \hline \multicolumn{1}{c}{ Year } & Source & $\begin{array}{l}\text { Size of } \\
\text { sample }\end{array}$ \\
\hline $\begin{array}{l}\text { Census years } \\
1900-1940\end{array}$ & Historical Statistics of the US (p. 20-21) & Whole population \\
1950,1960 & Historical Statistics of the US (p. 20-21) & 25\% sample \\
1970 & Historical Statistics of the US (p. 20-21) & 5\% sample \\
1980 & Census volume PC80-1-D1-A & Whole population \\
1990 & Census volume CP-1-1 & Whole population \\
2000 & Census table PCT007 on "FactFinder" & 20\% sample \\
2010 & Not recorded, replaced by ACS (see below) & \\
Inter-census years & & \\
$1901-1959$ & No data are available & $33,000-57,000$ \\
$1961-2004$ & Current Population Reports (CPR) & 2.5 million \\
$2005-$ & American Community Survey (ACS) on "FactFinder" & \\
\hline
\end{tabular}

Notes: The population samples used in the CPR are much too small to reflect widower populations under age 40 (see Table 3b). This means that before 2005 there are in fact no appropriate data for inter-census years. Incidentally, it can be observed that the data for marital status by age which are published in the annual volumes of the "Statistical Abstract of the United States" are identical to those published in the CPR (P20 Series). The only difference is that the age-group $17-18$ is omitted. This omission is probably motivated by the fact that for this age-group the estimates would be fairly poor. However, the comparisons performed in Table $3 \mathrm{~b}$ show substantial discrepancies even for older age groups up to $35-44$. "FactFinder" which is mentioned in the table refers to a search engine for statistical tables which is available on the website of the US Census Bureau.

immigration.

(2) The annual data about marriages and divorces are known to be fairly incomplete in some US states. Until 1996 total divorces were reported by the Federal Government. Subsequently, it ceased to publish national divorce data.

(3) Deaths which occur overseas are not included in the death numbers published by the US Census Bureau. In other words, the deaths of US soldiers in Europe, Korea, Vietnam, Afghanistan or Iraq were not included in annual death statistics.

It is true that fatality data are published by the Pentagon. However, such data are incomplete in two respects.

- Firstly, the Department of Defense does not publish official data for the fatalities among civilian contractors working for the armed forces. Whereas, during the Vietnam War the proportion of military personnel to civilian personnel was $6: 1$, during the occupation of Iraq it was almost $1: 1$ (Flounders 2009). In addition to the personnel under contract there are also persons who are not considered as contractors. For instance, one can mention news correspondents, businessmen, embassy personnel, Peace Corps affiliates, members of the Young Men's Christian Association (YMCA), and so on. When occurring abroad the deaths of such persons will be basically unrecorded. Young age groups will be particularly affected by such 
Table 3b: Percentage errors in various estimates of widower population.

\begin{tabular}{|c|c|c|c|c|c|c|c|c|}
\hline Year & Source & $\begin{array}{l}\text { Sample } \\
\text { size }\end{array}$ & & & & & & \\
\hline \multicolumn{9}{|l|}{1980} \\
\hline Age & & & $15-17$ & $18-19$ & $20-24$ & $25-29$ & $30-34$ & $35-39$ \\
\hline & Census & $100 \%$ & 992 & 1,089 & 5,970 & 11,759 & 16,531 & 22,337 \\
\hline & CPR & $0.02 \%$ & 0 & 0 & 2,000 & 8,000 & 11,000 & 19,000 \\
\hline 2000 & $\frac{\text { Census-CPR }}{\text { Census }}$ & & $100 \%$ & $100 \%$ & $66 \%$ & $32 \%$ & $33 \%$ & $15 \%$ \\
\hline Age & & & & $15-19$ & $20-24$ & $25-29$ & $30-34$ & $35-44$ \\
\hline & Census & $100 \%$ & & n.a. & n.a. & n.a. & n.a. & n.a. \\
\hline & Census & $20 \%$ & & 13,814 & 19,376 & 19,604 & 26,939 & 106,135 \\
\hline & CPR & $0.02 \%$ & & 3,000 & 0 & 9,000 & 15,000 & 96,000 \\
\hline 2010 & $\frac{\text { Census-CPR }}{\text { Census }}$ & & & $78 \%$ & $100 \%$ & $54 \%$ & $44 \%$ & $10 \%$ \\
\hline Age & & & $15-17$ & $18-19$ & $20-24$ & $25-29$ & $30-34$ & $35-39$ \\
\hline & Census & $100 \%$ & n.a. & n.a. & n.a. & n.a. & n.a. & n.a. \\
\hline & Census & $20 \%$ & n.a. & n.a. & n.a. & n.a. & n.a. & n.a. \\
\hline & ACS & $1 \%$ & 825 & 1,372 & 4,572 & 9,199 & 15,876 & 28,757 \\
\hline & ASES & $0.05 \%$ & 5,000 & 3,000 & 3,000 & 21,000 & 28,000 & 29,000 \\
\hline & $\frac{\text { ACS-ASES }}{\text { ACS }}$ & & $-506 \%$ & $-119 \%$ & $34 \%$ & $-128 \%$ & $-76 \%$ & $-1 \%$ \\
\hline
\end{tabular}

Notes: CPR means "Current Population Reports"; ACS means "American Community Survey"; ASES means "Annual Social and Economic Survey"; n.a. means "not available". The ratios (Census-CPR)/Census represent the errors in CPR estimates. The ratios (ACS-ASES)/ACS can also be seen as roughly representing the errors in ASES estimates. The sample size is given as a percentage of the total US population.

In the 2000 census the question of marital status was not asked on the short form sent to all people but only on the long form filled by about $20 \%$ of the population. In the census of 2010 the marital status question was not asked at all. It was replaced by the ACS, yet with lower accuracy due to a sample size which is only about $1 \%$ of the US population. Thus, surprisingly, over the past two decades census data about marital status by age have become less and less accurate. Incidentally, it can be observed that the CPR data are systematically below the census data which shows that the differences cannot solely be explained as being due to random sampling errors; there must also be a non-sampling error component.

Sources: Census 1980: Table 264 in the following census publication volume: US summary, Ch. D, Section A (available on the website of the US Census Bureau); CPR 1980: Series P-20, No 365, survey of March 1980 (issued in October 1981); Census 2000: Table PCT007 available on the FactFinder website of the US Census Bureau; CPR 2000: Series P-20, No 537 (issued in June 2001). ACS: Table B12002 available on the FactFinder website of the US Census Bureau; ASES 2010: "America's Families and Living Arrangements, Supplement", 2010. Many thanks to Dr. Rose Kreider from the US Census Bureau for her help.

omissions.

- Prior to 1980 the US Department of Defense did not publish worldwide fatality data. This point was made very clearly in 1993 when a data revision was announced by the Pentagon. Previously it had been said that 54,246 soldiers had died in the Korean War. According to the revision, there had been 36,516 deaths in Korea and 17,730 worldwide outside of the war theater. For the Vietnam War, published data tell us that there were 58,193 fatalities in the war theater but no data are available for 
the military fatalities outside the war theater.

In terms of magnitude, the total of omitted overseas deaths is certainly much smaller than the population of illegal immigrants.

In spite of these difficulties, computational methods are commonly used. For instance, in France population numbers by marital status and age were computed for every year from 1901 to 1993 (Daguet 1995). The main problem with such estimates is that it is impossible to control their quality. Usually, in such calculations one needs to make some assumptions, for instance that the death rate for an age group truly reflects the change that occurred in this age group. If for some reason (e.g. omitted overseas deaths) these assumptions are not correct then the result of the calculation will be biased. This is a non-sampling error which will not be removed by taking averages over several years (at least if the bias persists). The worst aspect is that researchers who use such calculated figures have no way whatsoever to control whether they are correct or not.

On the contrary, for population estimates based on samples, the resulting statistical uncertainty is well known and in addition it can be reduced by averaging over several years. Most of the data shown in this paper have been obtained in that way. The only exceptions are Fig. 1,2,7.

\section{Phase 1: Mid-age part of the Farr-Bertillon distribution}

Table $3 \mathrm{~b}$ shows that, except for middle age-groups, the accuracy provided by the "Current Population Reports" is fairly low especially for widowed persons. Another concern is the existence of a systematic non-sampling error component. In fact, we do not really know what population estimates were used to compute the death rates given in the "National Vital Statistics Reports" that were used in Fig. 2. The technical notes of the reports say only that "the populations used to calculate death rates were produced under a collaborative arrangement with the US Census Bureau". At least, this sentence suggests that the population estimates were not merely drawn from the CPR. Probably the CPR were used as a starting point and, in some (unspecified) way were corrected for small age-groups. Here the omission of the youngest age groups is a cautious and sensible step.

\section{Phase 2: The young widower effect}

In this section we present three sets of results.

- The first series of graphs (Fig. 3) is based on the censuses of 1940, 1950 and 1960. For these data the rates were computed by the US Department of Health and 

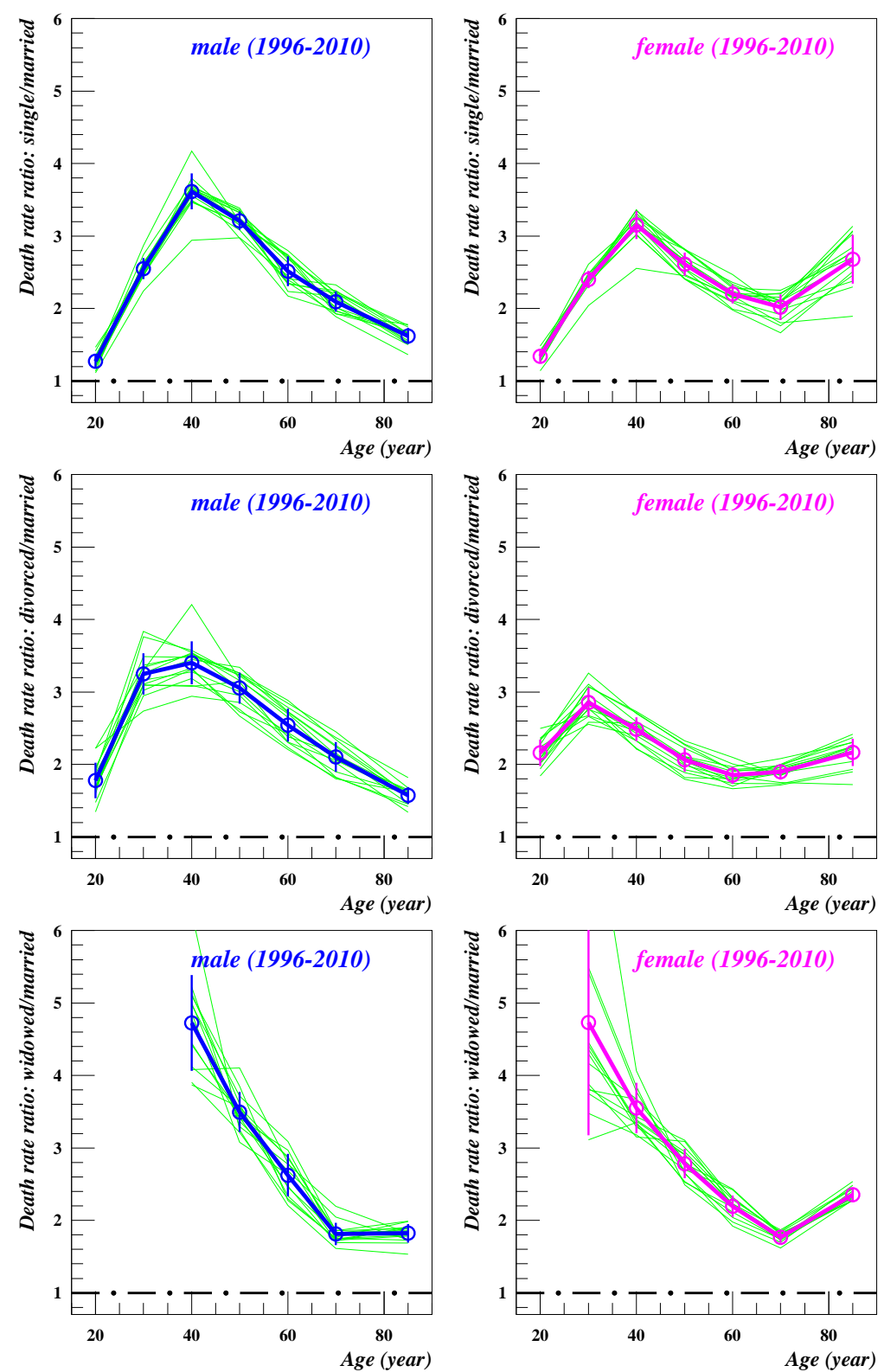

Fig. 2a,b,c: Death rate ratio according to marital status in the United States. From top to bottom: single/married, divorced/married, widowed/married. The thin (green lines) are the yearly curves for the 15 successive years. The thick lines show averages over the 15 years. During this period the ratios did not display any trend, there were only random fluctuations. There are 7 age groups ranging from $14-24$ to $64-75,>75$ but in the source no data are given for the youngest age groups of the $\mathrm{w} / \mathrm{m}$ case. In this graph as well as in all subsequent graphs the length of the error bars is $\pm 1.96 \sigma$ (where $\sigma$ is the standard deviation of the average) which corresponds to a probability confidence level of 0.95 . There are no data points for small age groups (particularly for young widowers/widows) because, apart from census years, there are no reliable estimates for the size of the corresponding populations. Source: National Vital Statistics Reports. Deaths: Final Data. Successive years from 1996 to 2010. National Center for Health Statistics. The publication gives the death numbers and the rates. How were these rates computed? The "Technical notes" attached to the table do not give any specific information. They say only that the population data "were produced under a collaborative arrangement with the US Census Bureau”.

published in the data compilation done by Robert Grove and Alice Hetzel (1968).

- The second series of graphs (Fig. 4) is based on the censuses of 1980, 1990 
and 2000. Actually in the census of 2000, marital status data were asked only on the so-called "long-form" which was distributed to $20 \%$ of the households.
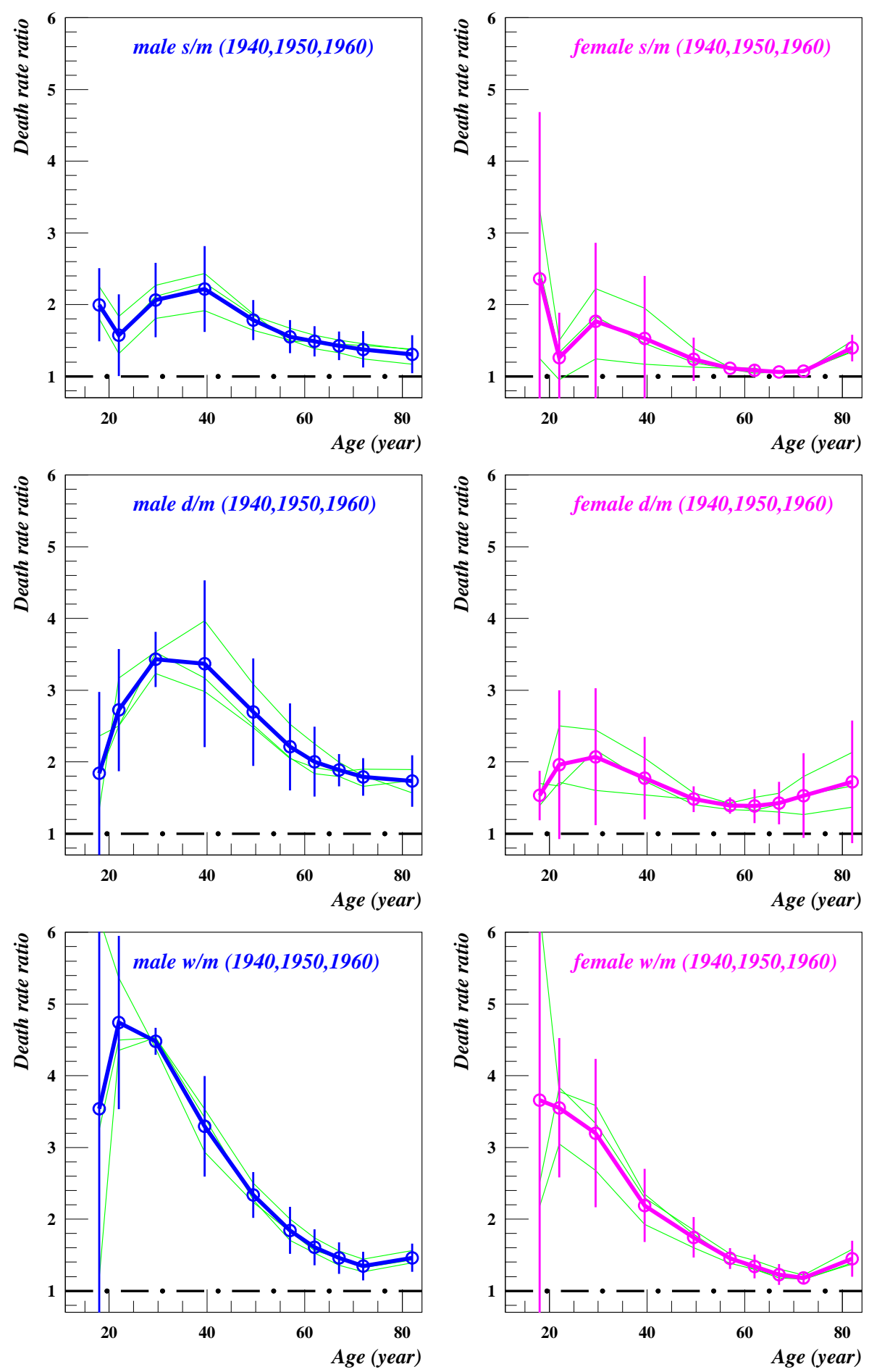

Fig. 3a,b,c: Death rate ratio with respect to the death rate of married persons: United States, 1940, 1950, 1960. From top to bottom: single/married, divorced/married, widowed/married. The thin (and green) curves are for each of the 3 years while the thick lines with the round dots show their average. There are 10 age groups: < 20, $20-24,25-34,35-44,45-54,55-50,60-64,65-69,70,74,>75$ As expected, the error bars become fairly large for small age groups, particularly for young widowers and widows. Source: Grove and Hetzel (1968, p.334). The publication gives directly the rates. 

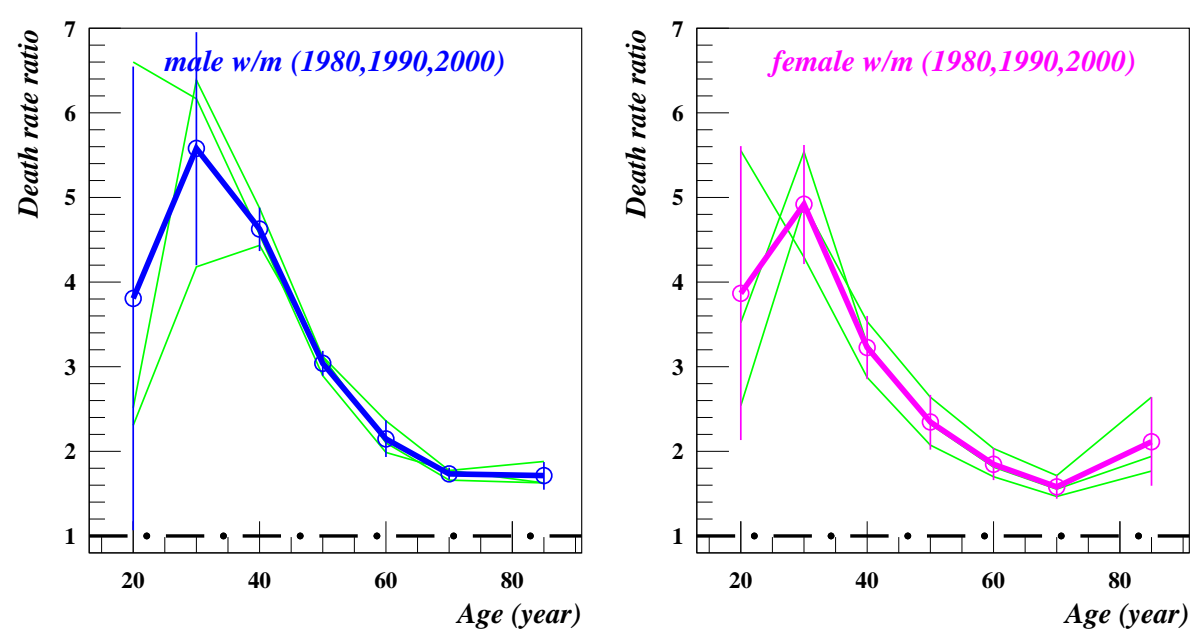

Fig. 4: Death rate ratio for widowed persons (i.e. their death rate divided by the death rate of married persons), United States, 1980, 1990, 2000. The thin (and green) curves are for each of the 3 years while the thick lines with the round dots show their average. The age groups are the same as in Fig. 2. As expected, the error bars become fairly large for young widowers and widows.

Sources: Census population data: 1980: Vol. PC80-1-D1-A (Table 264, p. 1-67); 1990: General population characteristics, Vol. CP-1-1, (Table 34, p. 45); 2000: "American FactFinder" website of the US Census Bureau, Table PCT007.

Mortality data: 1980: Vital Statistics of the United States 1980, Vol. 2, part A, Table 1-31 (p. 315); 1990: Vital Statistics of the United States 1990, Vol. 2, part A, Table 1-34 (p. 387); 2000: National Vital Statistics Report, Vol. 50, No 15, 16 Sep 2002.

- In Fig 3 and 4 the error bars for widowed persons in the young age groups remain very large. In an attempt to reduce them, we use data for 6 successive years. These data are based on the "American Community Survey" which is answered by about $1 \%$ of the households. Thanks to the 6-year interval, the error bars are notably reduced.

\section{Error bars}

As stated in the caption of Fig. 2, the length of the error bars are $\pm 1.96 \sigma$ (average). The standard deviation of the average was computed by dividing the standard deviation of $n$ annual curves $Y_{j}, j=1, \ldots, n$ by the standard $1 / \sqrt{n}$ factor. However, this factor is correct only when the $Y_{j}$ are not correlated. While there is indeed a low correlation for young age groups, for older age groups there is an average correlation of $r_{m}=0.90$. For these data points the factor $1 / \sqrt{n}$ should be replaced by $f=\sqrt{1+(n-1) r_{m}} / \sqrt{n}$ (Roehner 2007, p. 45). With $n=6$ and $r_{m}=0.90$ the factor $f$ is almost equal to 1 . In other words, except for young age groups, the error bars shown in the graph underestimate the actual confidence intervals. On the other hand, using the factor $f$ everywhere would result in overestimating the confidence interval for young age groups, the only ones which really matter in this respect. 

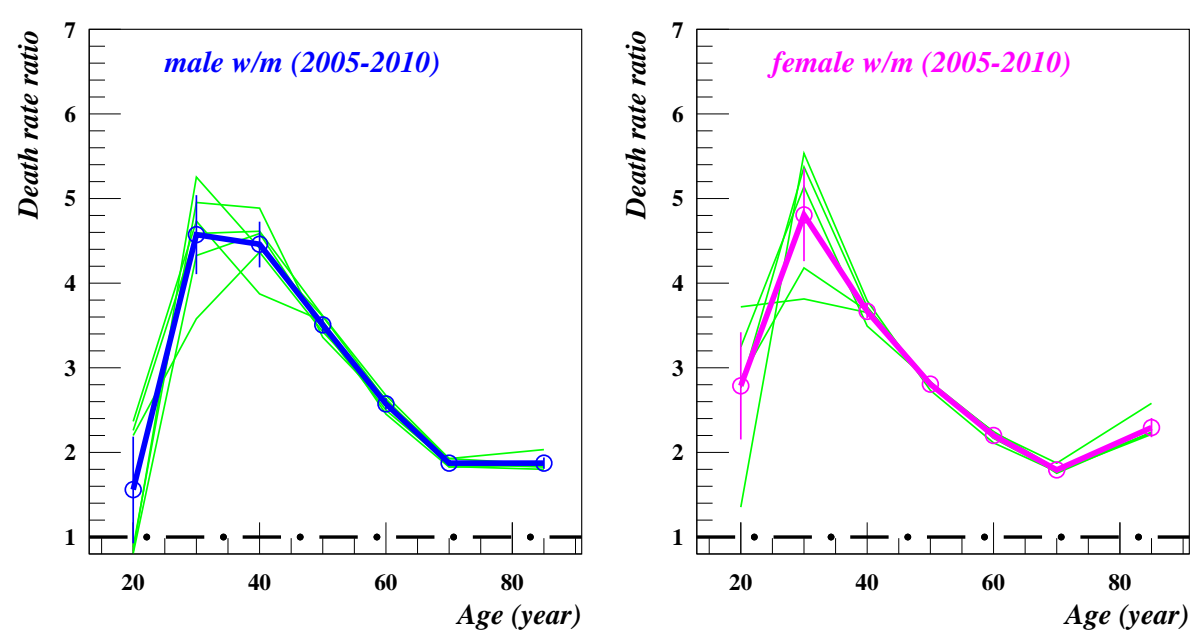

Fig. 5: Death rate ratio of widowed persons, United States, 2005-2010. The thin (and green) curves are for the 6 successive years while the thick lines with the round dots show their average. The age groups are the same as in Fig. 2. The length of the error bars is $\pm 1.96 \sigma$ which corresponds to a probability confidence level of 0.95. The data used in this graph for the populations are not census data but are based on samples of about 2.5 million respondents. This "experiment" confirms the existence of a dip for the youngest age group $15-24$. Sources: Populations: Starting in 2005 data by marital status and age are provided by the "American Community Survey" and are made available on the "American FactFinder" website set up by the US Census Bureau (Table B12002). Mortality data: National Vital Statistics Reports entitled "Deaths: Final Data”. In 2015 the most recent year available was 2010.

\section{What is the influence of cohabitation and separated couples?}

In recent decades the traditional picture of family life has become more complicated due to the following trends.

(1) In 1960, 72\% of all American adults were married; in 2012 just $50 \%$ were.

(2) During the same time interval, the number of cohabiting non-married couples of opposite sex jumped from $1.1 \%$ to $11 \%$. Note that because different states may not have the same definition of cohabitation the last percentage may be subject to an error margin of about $\pm 10 \%$.

(3) Finally around 2005, in about $8 \%$ of married couples one of the spouses was not present. In 1980 this proportion was about $6 \%$.

Needless to say, such trends are by no means special to the United States; they are shared by many other western countries. However, the trends are perhaps more surprising in the United States because traditionally this country has put a strong social emphasis on family life. In this respect it can be recalled that in 2014 three states, Mississippi, Michigan, and Florida, still had laws against cohabitation by oppositesex couples.

How do the previous trends affect the interpretation of our results?

We will successively consider the effects of cohabitation and separation 


\section{Implication of cohabitation}

In itself point 1 will not affect our results but in fact it is strongly connected with the second point: those people who do not get married are doing so because they are living together without being married.

To make the argument clearer let us make the following simple assumptions. We assume that the real death rates in the married, single and widowed classes are 1, 2 and 3 per 1,000. In addition we assume that $50 \%$ of the persons registered as single or as widowed are in fact cohabiting with a partner. For the sake of simplicity we assume that there are 2,000 single and 2,000 widowed persons. Moreover we assume that the measured death rate of married people is not modified and is equal to the real death rate. This makes sense as long as the number of cohabiting people remains small compared to married people.

Under these assumptions, what are the death rates, $d_{m e}$, and death rate ratios, $r_{m e}$, that will be measured and how do they compare to the real death rate ratios $r_{r e}$ ?

- Death rate ratio of single persons

$$
d_{m e}(s)=(2+1) / 2=1.5 \quad \rightarrow \quad r_{m e}(s)=1.5 / 1=1.5, \quad \text { vs. } r_{r e}(s)=2 / 1=2
$$

\section{- Death rate ratio of widowed persons}

$$
d_{m e}(w)=(3+1) / 2=2.0 \quad \rightarrow \quad r_{m e}(w)=2.0 / 1=2.0, \quad \text { vs. } r_{r e}(w)=3 / 1=3
$$

In other words, due to cohabitation our measurements will underestimate the actual death rate ratios of single, and widowed persons. The same argument applies of course to divorced persons.

\section{Implication of separation}

In the present argument we suppose that there is no cohabitation which means that the death rates of single and widowed persons are correct. In addition, in the same way as above, we assume that $50 \%$ of the married persons are in fact separated. With the same real death rates as above what will be the measured death rate ratios? Whereas previously, the numerators of the death rate ratios were affected, this time the denominators are affected.

\section{- Death rate ratio of single persons}

$d_{m e}(m)=(1+2) / 2=1.5 \quad \rightarrow \quad r_{m e}(s)=2 / 1.5=1.33, \quad$ vs. $r_{r e}(s)=2 / 1=2$

- Death rate ratio of widowed persons

$d_{m e}(m)=(1+2) / 2=1.5 \quad \rightarrow \quad r_{m e}(w)=3.0 / 1.5=2.0, \quad$ vs. $r_{r e}(w)=3 / 1=3$

In other words, separation will also make our measurements to underestimate the actual death rate ratios. 
Because the two effects go in the same direction, their combination should also result in underestimating the real death rate ratios. An additional conclusion is that if we see a weakening of the Farr-Bertillon effect over the coming decades it may well be a statistical artifact due to persistent cohabitation and separation trends.

\section{Death ratios by marital status and age for selected causes}

In this section we consider death rate ratios according to selected causes of death. Previously we have seen that the death ratios for never-married and divorced persons are somewhat similar. Therefore, the present investigation will be restricted to nevermarried and widowed persons.

The main difficulty with data by cause of death is the fact that the death numbers are fairly small which creates large fluctuations. In an attempt to smooth them out as far as possible we lumped together not only successive years (as was already done in previous graphs) but also the two genders.

Incidentally, it can be noted that death numbers by cause of death, marital status and age exist from 1979 to 1993 , but only 1980 and 1990 can be used because for the populations we must rely on census data.

How well do the 6 selected causes of death represent all causes? For married persons, in the age-group 15-24 the cumulative death rates (per 100,000) of the 6 selected causes total 51 (average of 1980 and 1990); that is only slightly less than the total for all causes which is 74 .

The graphs in Fig. 6 can help us to better understand the origin of the fluctuations observed in the left-hand side of the curves for widowed persons. For convenience we denote the first two data points of these curves by $r_{1}$ and $r_{2}$. In some graphs $r_{1}$ was higher than $r_{2}$, whereas in others it was the opposite. Why? In order to connect the aggregated curves of Fig. 4-5 with those displayed in Fig. 6 we must first ask ourselves what are the leading causes of death. In the age-group $15-24$ the main factors are motor vehicle accidents with a rate of 31 per 100,000 (average rate for married persons in 1980 and 1990), followed by suicide with a rate of 11 and cancer with a rate of 5.1. Thus, in this age-group, motor vehicle accidents represent almost one half of the total death rate. Despite this particular factor being larger than all the others, it has huge fluctuations. Indeed, the error bars for motor vehicle accidents are much broader than for cancer or heart disease for which death numbers are much smaller.

In short, it appears that the uncertainty affecting the youngest age groups is due to the very high volatility assocated with motor vehicle death statistics. 

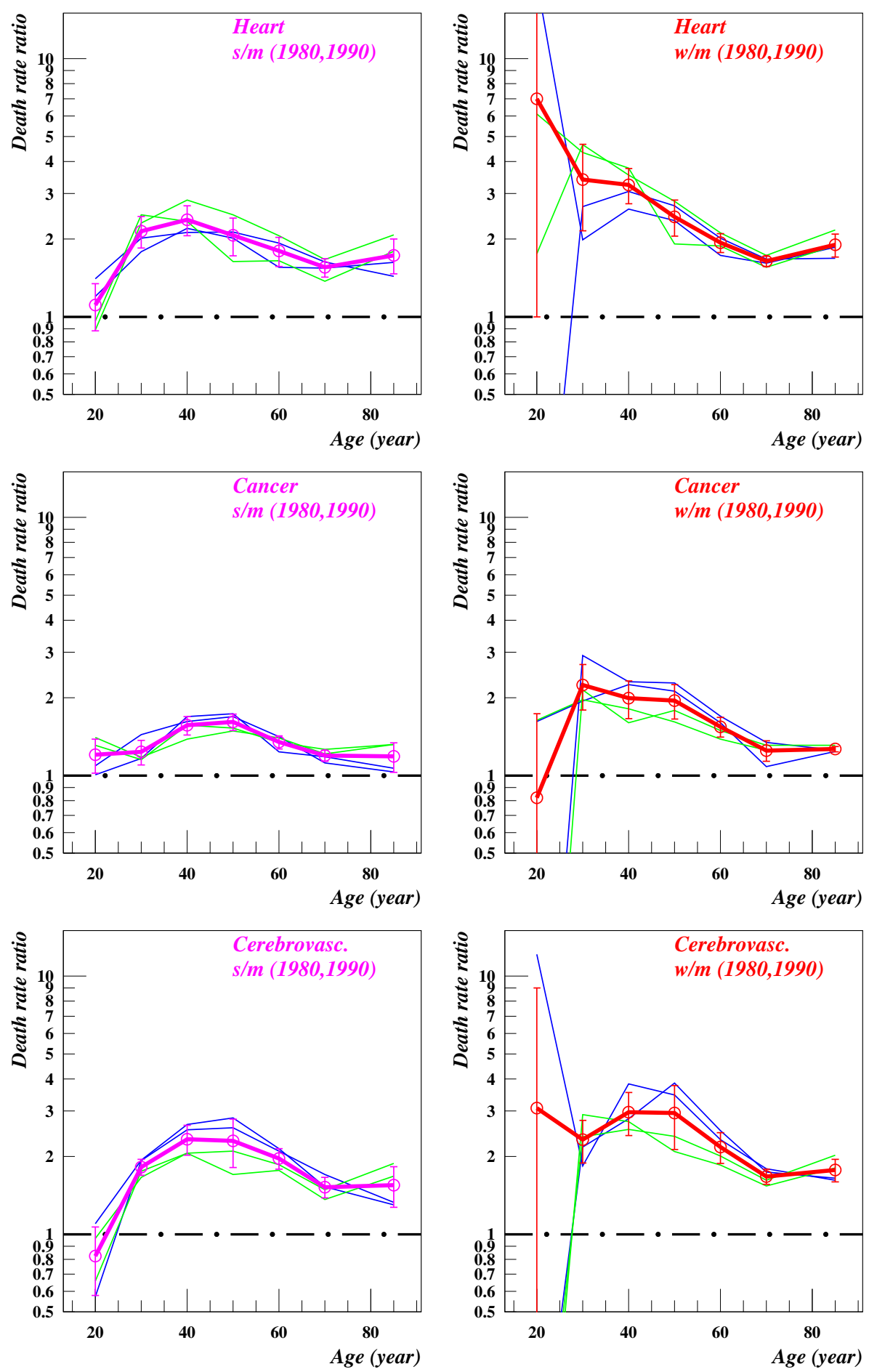

Fig. 6a,b,c: Death rate ratio for different causes of death, United States. The $s / m$ graphs on the left-hand side are for single/married, whereas the $w / m$ graphs are for widowed/married. The graphs show 1980 and 1990 data for males (thin blue lines) and for females (thin green lines). The thick lines represent the averages of the 4 series. The age groups are the same as in Fig. 2. Sources: 1980: Vital Statistics of the United States, 1980, Vol. 2, Part A, table 1-31, p. 315-324. 1990: Vital Statistics of the United States, 1990, Vol. 2, Part A, table 1-34, p. 387-400. In order to compute the rates we used census population data which is why the analysis is restricted to census years.

Because almost all death ratios documented in Fig. 6 are larger than 1 it makes sense to consider averages over all age groups. This will allow a ranking of the causes of 

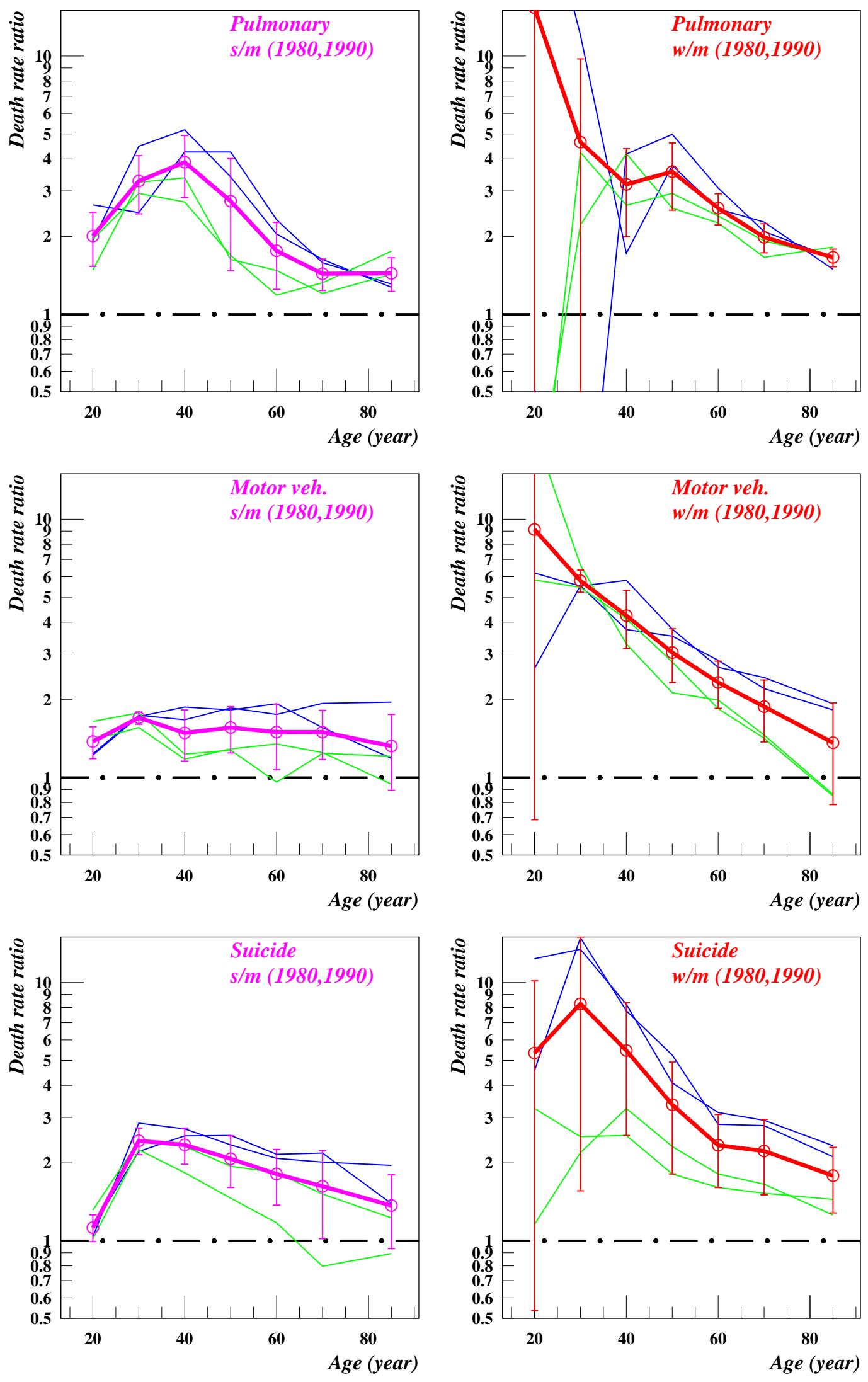

Fig. 6 d,e,f: Death rate ratio for different causes of death, United States (continued). The comments made in Fig. 6 a,b,c apply as well to the present graphs. Sources: Same as for Fig. 6 a,b,c 
death according to their death ratios (Table 4).

Table 4: Ranking of causes of death according to their average death ratio

\begin{tabular}{|c|c|c|c|c|c|c|}
\hline Marital status & & & & & & $\begin{array}{l}\text { Average } \\
\text { (all } 5 \text { causes) }\end{array}$ \\
\hline \multicolumn{7}{|l|}{ Never married } \\
\hline & suicide & heart & cerebrovasc. & motor veh. & cancer & \\
\hline Death ratio s/m & $1.8 \pm 0.4$ & $1.8 \pm 0.3$ & $1.76 \pm 0.4$ & $1.5 \pm 0.1$ & $1.3 \pm 0.1$ & $1.6 \pm 0.1$ \\
\hline \multicolumn{7}{|l|}{ Widowed } \\
\hline & suicide & motor veh. & heart & cerebrovasc. & cancer & \\
\hline Death ratio w/m & $4.1 \pm 1.7$ & $4.0 \pm 2$ & $3.1 \pm 1.4$ & $2.4 \pm 0.7$ & $1.6 \pm 0.4$ & $3.0 \pm 0.4$ \\
\hline \multicolumn{7}{|c|}{$\begin{array}{l}\text { Notes: The figures given in this table are averages over the } 7 \text { age groups considered in Fig. } 6 \text {. Overall the death } \\
\text { ratio for w/m is about twice the death ratio of } \mathrm{s} / \mathrm{m} \text {. However, with the exception of motor vehicle accidents, } \\
\text { the ranking is almost the same. The error bars are for a probability confidence level of } 0.95 \text {. The "pulmonary } \\
\text { disease" cause of death has not been included in this ranking because it has very large fluctuations: its coeffi- } \\
\text { cients of variation are } 40 \% \text { for s } / \mathrm{m} \text { and } 102 \% \text { for w/m. The } 6 \text { causes of death under consideration correspond } \\
\text { to the following code numbers in the } 9 \text { th Revision of the International Classification of Diseases of } 1975 \text { : heart } \\
\text { diseases: } 390-398+404-429 \text {, cancer: } 140-208 \text {, cerebrovascular diseases: } 430-438 \text {, pulmonary diseases: } \\
490-496 \text {, motor vehicle accidents: E } 810-E 825 \text {, suicide: E950-E959. } \\
\text { Sources: Same sources as for Fig. } 6\end{array}$} \\
\hline
\end{tabular}

Because the $\mathrm{w} / \mathrm{m}$ death ratios are based on smaller population numbers than the $\mathrm{s} / \mathrm{m}$ ratios they have higher volatility. Nevertheless, the fact that the ranking of causes of death is almost the same in the two cases shows that overall the $\mathrm{w} / \mathrm{m}$ ratios are trustworthy.

\section{Marital ties as a most effective drug}

Can marriage be considered as an effective multipurpose drug? Yes and no.

"No" for a very obvious reason: it can only reduce the death rates of persons who are not already married. What proportion do non-married persons (to be distinguished from the never-married) represent in the age-group 65-74? In 2005 for instance, according of the data provided by the "American Community Survey" the non-married were $21 \%$ for men and $43 \%$ for women.

The previous question can also be answered affirmatively because for single or widowed persons, marriage makes really a big difference. In this respect, one should remember that in clinical test trials most pharmaceutical drugs, for instance those against heart disease, provide at most a 20\% - 30benefit (more details can be found in Roehner 2014). On the contrary, Fig. 6 shows that except for cancer in old age, the death rate is divided at least by a factor 1.5, which represents a reduction of $33 \%$, and in many cases the reduction is over $50 \%$ (division by 2 ). 


\section{Suicide}

\section{Why is suicide of special interest?}

Among the causes of death considered previously, suicide has a special significance for (at least) four reasons.

- Historically, since the mid 19th century, the phenomenon of suicide arose considerable interest among sociologists. The work of Emile Durkheim (1897) is probably the most well known but there were many other studies, for instance by LouisAdolphe Bertillon and his son Jacques Bertillon.

- Durkheim showed that persons with many family links have smaller suicide rates. For married persons with respect to never-married or widowed persons, this connection was already well-known before Durkheim. Although the influence of the number of children had also been observed (particularly by Bertillon, see above), the relationship was not known accurately because of the fact that death certificates did not contain information about the number of children. Durkheim was able to establish the existence of a negative correlation between number of children and suicide rates by taking advantage of regional differences in suicide rates on the one hand and in average number of children by household on the other hand. This observation (largely forgotten nowadays) was a strong argument in favor of Durkheim's thesis of a connection between the strength of family ties and low suicide rates.

- In biology a phenomenon called apoptosis is often referred to as "cell suicide". Apoptosis occurs when cells no longer receive "stay alive" signals from their neighbors. More details can be found in Raff (1998), in chapter 12 of Roehner (2007) and in Wang et al. (2013). This link with apoptosis gives at least a plausible mechanism for the connection between suicide and interaction with nearest neighbors. For other causes of death we do not have even the beginning of an understanding.

- Finally, a look at Fig. 6 and Table 4 shows that suicide is the cause of death for which the death ratios are highest.

\section{Suicide ratios in France (1968-1993)}

In Fig. 6f we have fairly large statistical fluctuations. As always in such a situation, we wish to reduce them. There is only one way to do that: one must increase the numbers of the events. This means either increasing the size of the country or increasing the number of years. Here we adopt the second approach. We consider France, which is smaller than the United States, but for which data over a period of 26 years can be obtained. The population of France is about 5 times smaller than the US population but with respect to the graphs displayed in Fig. $6 f$ we will gain a factor: $(26 / 2) / 5=2.6$. In addition, shifting from the US to another country will tell us something about the robustness of the Farr-Bertillon effect. 

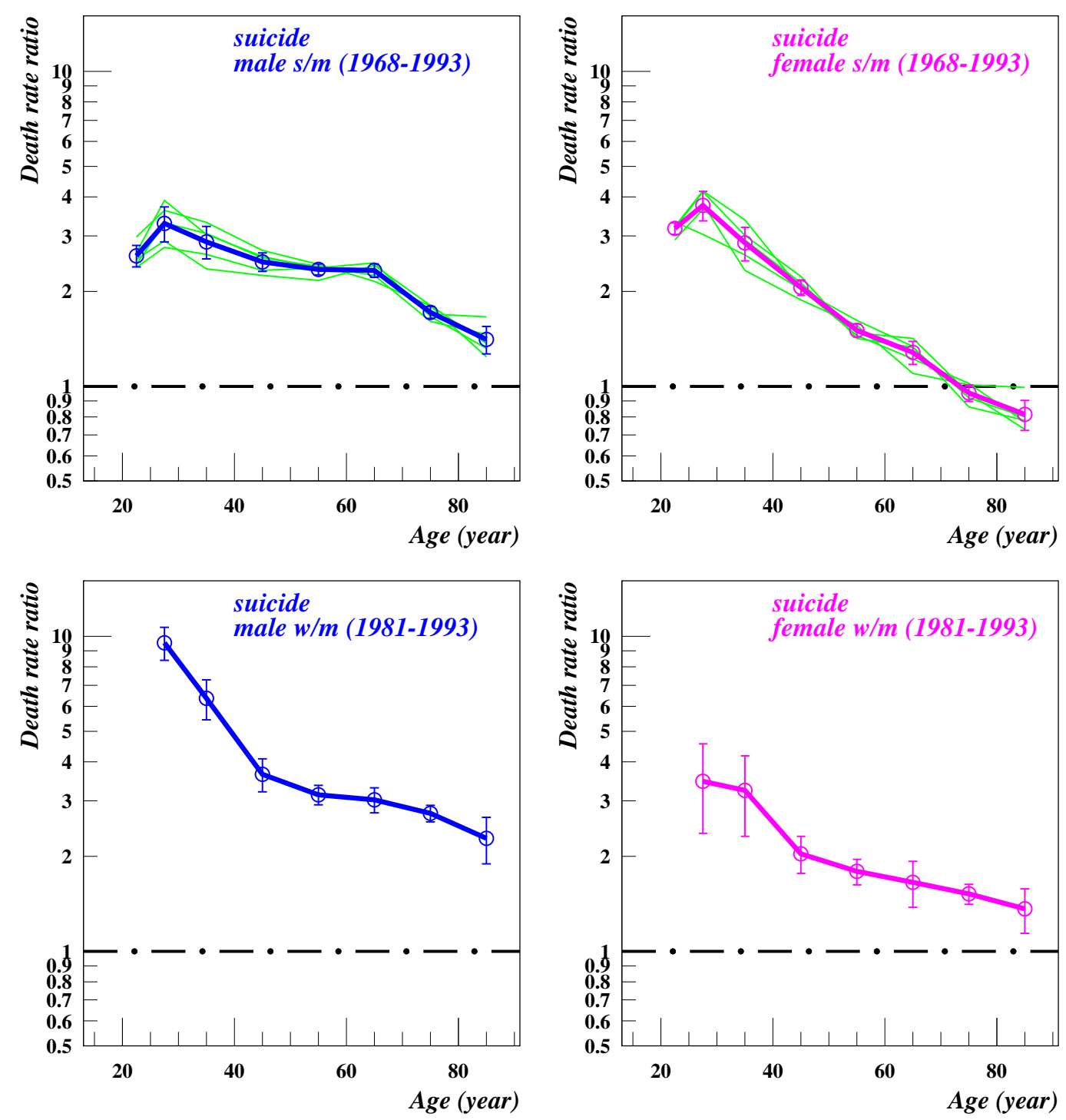

Fig. 7a,b: Death rate ratio for suicide in France. The age groups are: $20-24,25-29,30-39,40-49,50-$ 59, 60 - 69, 70 - 79, > 80. Source: Besnard (1997, p. 744, 752)

Regarding the accuracy of the present data set we must also ask ourselves how the populations of the age-groups by marital status have been estimated. For inter-census years they were estimated through a computational procedure whose results were published in Daguet (1995). As already observed, the correctness of such a procedure can hardly be checked.

\section{Conclusions and perspectives}

The Farr-Bertillon effect is not yet well recognized as a major determinant of death rate. This is particularly true for medical doctors and is attested by the fact that the characteristics of patients taking part in medical trials include many parameters, yet their marital status is usually not reported in spite of the fact that it will substantially affect the outcome of the trial. More details on this point can be found in Roehner 
(2014).

\section{Conclusions}

Our investigation of the Farr-Bertillon effect led to the following conclusions.

- In spite of the important demographic and sociological changes that took place over the past century the Farr-Bertillon effect remained fairly unchanged.

- The death rate ratios computed from different data sets (based on censuses or surveys) are well consistent with one another.

Table 5 Summary of death rate ratios $w / m$ computed in the present paper, USA, 1940-2010

\begin{tabular}{|c|c|c|c|c|c|}
\hline & $\begin{array}{l}\text { Male } \\
\text { or } \\
\text { Female }\end{array}$ & Age & $\begin{array}{c}\text { Average } \\
1940-1960\end{array}$ & $\begin{array}{c}\text { Average } \\
1990-2010\end{array}$ & $\begin{array}{r}\text { Coefficient } \\
\text { of } \\
\text { variation } \\
\text { for } 1990-2010\end{array}$ \\
\hline & \multicolumn{5}{|l|}{ Male } \\
\hline & & 40 & 3.2 & 4.5 & $2.6 \%$ \\
\hline & & 50 & 2.3 & 3.3 & $7.9 \%$ \\
\hline & & 60 & 1.7 & 2.5 & $12.0 \%$ \\
\hline \multicolumn{6}{|c|}{ Female } \\
\hline & & 40 & 2.2 & 3.5 & $5.7 \%$ \\
\hline & & 50 & 1.8 & 2.7 & $8.6 \%$ \\
\hline & & 60 & 1.4 & 2.5 & $11.1 \%$ \\
\hline $\begin{array}{l}\text { Notes: The tables sumn } \\
5 \text { for 1990-2010. The c } \\
\text { various sources. The a } \\
\text { over past decades seen } \\
\text { European countries can } \\
\text { which bring about such }\end{array}$ & $\begin{array}{l}\text { arizes the } \\
\text { efficient o } \\
\text { e column } \\
\text { ere is also } \\
\text { e found in } \\
n \text { increase }\end{array}$ & $\begin{array}{l}\text { death } \\
\text { f varia } \\
\text { gives t } \\
\text { obser } \\
\text { Vallir }\end{array}$ & $\begin{array}{l}\text { ate ratios } w / \\
\text { tion, that is t } \\
\text { he middle of } \\
\text { ved in Europ } \\
\text { et al. (2001 }\end{array}$ & $\begin{array}{l}m \text { computed } \\
\text { say } \sigma / m, g \\
\text { the } 10 \text {-year } \\
\text { e; the death } \mathrm{r} \\
\text { p. } 318-321)\end{array}$ & $\begin{array}{l}\text { in Fig. } 3 \text { for } 1940-1960 \text { and in Fig. } 2,4 \text {, } \\
\text { ives an estimate of the fluctuations due to } \\
\text { intervals of the age groups. The increase } \\
\text { ate ratios }(\text { not - married }) / m \text { in several } \\
\text { We do not yet know what are the factors }\end{array}$ \\
\hline
\end{tabular}

The results summarized in Table 5 are restricted to the age interval 40-60 because Fig. 2 is limited to this interval.

- Whereas for single and divorced persons the death rate ratios are bell-shaped with a maximum around the age of 40 , for widowed persons it is a function which either decreases steadily from youngest to oldest age groups or which has a maximum at the second youngest age interval. It is this last shape which is found in the observations of highest quality. However, we have seen that the shape of the curve for young age groups is mainly determined by the trafic accidents, a component which may be modified by changes in trafic regulation rules.

- When different causes of death are investigated it appears that suicide leads to the highest average death rate ratio while cancer leads to the lowest.

- In recent decades death rate ratios have been increasing in Europe as well as in the United States. We do not yet know what is the reason of such an increase. May be it is a consequence of cohabitation without marriage which has become so 
widespread in the US as in Europe but it is not clear how the two phenomena are related. It should be observed that it is not only $w / m$ which is increasing but also $s / m$. As cohabitation makes the condition of "singles" (in mame) very similar to the condition of married persons one should observe a convergence od $d_{s}$ toward $d_{m}$ that is to say a convergence of $d_{s} / d_{m}$ toward 1 .

\section{Extraction of the dynamical response}

In fact, demographic statistics of the kind considered in the present paper give little information about the dynamical aspect of this phenomenon. We learned that "on average" for 10-year age-groups, widowers have a death rate which is 3 times the death rate of married persons. However, this observation does not tell us anything about the transition from one state to another. How long does it take? Does the death rate of recent widowers increase steadily toward a steady state or is there a shock effect during which the death rate ratio first overshoots its steady limit?

One way to answer this question is to follow a sample of married persons over several years. This was done in a number of studies: Bojanovsky $(1979,1980)$, Frisch et al. (2013), Helsing et al. (1981), Mellström et al. (1982), Parkes et al. (1969), Thierry (1999, 2000), Young et al. (1963). Needless to say, in order to observe a substantial number of deaths in a sample of married people followed by a sizable number of deaths of widowers one should work on a sample of elderly persons. That is why most of the previous studies concern persons over 50 or 60 . Basically, they found that the death rate of widowers peaked in the first 3 or 6 months after widowhood and then returned to the rate of married persons.

How can one connect this observation to the death rate ratios measured in the present paper? Most of our observations concerned 10-year age groups? Such age groups will contain a mixture of widowers who differ both in age $(a)$ and in the length of widowhood time $(w)$.

Through the studies mentioned above, we know that the death rate of widowers $(d)$ will be a function not only of $a$ but also of $w: d=d(a, w)$. Similarly, the remarriage rate of widowers $\left(d^{\prime}\right)$ will also be a function of $a$ and $w: d^{\prime}=d^{\prime}(a, w)$. Because age is recorded on death and marriage certificates, one knows how, for fixed $w, d$ and $d^{\prime}$ depend upon $a$. Unfortunately, because $w$ is not recorded on death and marriage certificates, there is no direct information about how $d$ and $d^{\prime}$ depend upon $w$ (for fixed $a$ ). The functions $d_{a}(w), d_{a}^{\prime}(w)$ summarize the dynamic responses of a group of widowers of age $a$.

The objective of the second part of this study will be to extract these functions from the data describing the Farr-Bertillon effect. To say it in a different way, from the death rate ratio per age-group $r_{g}=W_{g} / M_{g}$ we wish to extract $r(w)$, the instanta- 
neous death rate ratio of a cohort who entered widowhood at the same moment, as a function of widowhood length.

While being a well defined objective, it is not an easy one. The solution that we will propose in Part 2 of this study may not be the only one possible, nor may it be the simplest or most satisfactory. That is why we hope that the present paper will raise the interest of other researchers. We welcome in advance any progress in this direction by other groups. That is also why the present paper has an Appendix which provides the primary data of those of our observations that can be considered as the most accurate, namely the three stars observations of Table 2 . Those data should permit to test theoretical models.

Acknowledgments The authors wish to express their gratitude to Drs. Jason Fields and Rose Kreider of the US Census Bureau, and to Dr. Betzaida Tejada Vera of the Center for Diseases Control. Their advice helped us to locate the most accurate data available.

\section{Appendix: Death rates by age, marital status and sex}

Table A1: Death rates by marital status and age, USA, census years: 1940, 1950, 1960

\begin{tabular}{lccccccccccc}
\hline \hline & $<20$ & 20 & 25 & 35 & 45 & 55 & 60 & 65 & 70 & $>75$ \\
& & - & - & - & - & - & - & - & - & \\
& & 24 & 34 & 44 & 54 & 59 & 64 & 69 & 74 & \\
\hline Men, death rate & & & & & & & & & & \\
Single, 1940 & 4.5 & 2.9 & 4.7 & 9.2 & 17.4 & 28.7 & 38.3 & 52.3 & 75.1 & 132 \\
Single, 1950 & 3.3 & 2.2 & 3.6 & 8.3 & 17.2 & 29.6 & 40.8 & 55.0 & 79.5 & 137 \\
Single, 1960 & 2.7 & 2.2 & 3.4 & 7.3 & 15.7 & 23.7 & 38.0 & 53.8 & 76.3 & 138 \\
Married, 1940 & 2.5 & 2.2 & 2.6 & 4.8 & 10.6 & 19.1 & 27.6 & 39.4 & 60.3 & 113 \\
Married, 1950 & 1.7 & 1.4 & 1.7 & 3.6 & 9.3 & 17.7 & 25.9 & 36.6 & 54.6 & 100 \\
Married, 1960 & 1.2 & 1.2 & 1.5 & 3.0 & 8.4 & 16.0 & 25.3 & 37.2 & 53.4 & 100 \\
Widowed, 1940 & 15.5 & 11.8 & 11.4 & 14.1 & 23.7 & 34.8 & 43.1 & 57.4 & 79.7 & 162 \\
Widowed, 1950 & 2.0 & 6.1 & 7.7 & 12.3 & 21.1 & 30.2 & 39.6 & 49.7 & 69.1 & 139 \\
Widowed, 1960 & 3.9 & 5.4 & 6.8 & 10.6 & 21.0 & 32.0 & 44.0 & 57.9 & 77.0 & 156 \\
\hline
\end{tabular}

Notes: The rates are given in deaths per 1,000 population of specified group. Separate data for death numbers and populations are not available. It can be observed that, in accordance with Gompertz law (Gompertz 1825), the death rates increase exponentially with age with a doubling time of about 10 years.

Source: Grove and Hetzel (1968, p. 334)

This Appendix gives the death numbers and population data for US census years from 1940 to $2000^{5}$. Although, most of these data are available on Internet, they are

\footnotetext{
${ }^{5}$ The regular publication of death data by age, marital status and sex began in 1979. However, because marital status was recorded on death certificates, Grove and Hetzel (1968) from the US Census Bureau were able to compute and publish death rates for the census years 1940, 1950 and 1960. That is why no death numbers are available for these years. For 1970 there neither death rates nor death numbers.
} 
Table A2: Death and population by marital status and age, USA, census years: 1980, 1990, 2000

\begin{tabular}{lrrrrrrr}
\hline & $15-24$ & $25-34$ & $35-44$ & $45-54$ & $55-64$ & $65-74$ & $>75$ \\
\hline Men, deaths & & & & & & & \\
Single, 1980 & 30799 & 13739 & 6463 & 10154 & 16251 & 20804 & 25702 \\
Single, 1990 & 24921 & 25448 & 19324 & 11609 & 14492 & 18973 & 26611 \\
Single, 2000 & 21247 & 16615 & 21731 & 20464 & 15203 & 18119 & 28299 \\
Married, 1980 & 4894 & 16144 & 23238 & 57550 & 134153 & 199962 & 210401 \\
Married, 1990 & 2386 & 12868 & 24234 & 43802 & 105347 & 195495 & 257631 \\
Married, 2000 & 1493 & 8218 & 22160 & 49298 & 87351 & 164106 & 312544 \\
Widowed, 1980 & 76 & 223 & 539 & 2704 & 12656 & 38900 & 130987 \\
Widowed, 1990 & 29 & 218 & 619 & 1852 & 9329 & 35630 & 143605 \\
Widowed, 2000 & 49 & 148 & 656 & 2125 & 7278 & 30800 & 182316 \\
Men, Population & & & & & & & \\
Single, 1980 & 17723 & 4409 & 978 & 657 & 551 & 364 & 198 \\
Single, 1990 & 16516 & 7779 & 2493 & 838 & 555 & 392 & 225 \\
Single, 2000 & 17450 & 7791 & 4071 & 1783 & 548 & 385 & 247 \\
Married, 1980 & 3424 & 12589 & 10488 & 9365 & 7716 & 5496 & 2329 \\
Married, 1990 & 2089 & 12183 & 13710 & 9863 & 8205 & 6400 & 3156 \\
Married, 2000 & 2331 & 10797 & 15890 & 13747 & 9131 & 6581 & 4200 \\
Widowed, 1980 & 8.05 & 28.2 & 53.3 & 152 & 366 & 602 & 891 \\
Widowed, 1990 & 10.1 & 32.3 & 71.8 & 134 & 346 & 701 & 1079 \\
Widowed, 2000 & 28.3 & 120 & 304 & 665 & 1776 & 3587 & 5637 \\
\hline
\end{tabular}

Notes: The numbers of deaths are expressed in units while the populations are in thousands. The data given in the table are the primary data from which the death rate ratios $s / m$ and $w / m$ were computed. The data for females can be drawn from the same sources; they were omitted here in order to save space.

Sources: Same as for Fig. 4.

not easy to locate. For instance after 1970, census population data are buried among dozens of volumes and thousands of pages of census publications; this makes their identification and extraction fairly time consuming.

\section{References}

The comments at the end of some of the entries will be removed in the final version of the paper. They focus on how those works contribute to our present investigation.

Accuracy of data on population characteristics as measured by CPS-census match, 1960. Series ER 60, No 5. Bureau of the Census. US Government Printing Office, Washington DC.

Bertillon (L.-A.) 1872: Article "Mariage" in the Dictionnaire Encyclopédique des Sciences Médicales, [Encyclopedic Dictionary of the Medical Sciences]. 2nd series, Vol. 5, p.7-52.

[Available on "Gallica", the website of digitized publications of the French na- 
tional library, at http://www.bnf.fr]

Bertillon (L.-A.) 1879: Article "France" in the Dictionnaire Encyclopédique des Sciences Médicales, [Encyclopedic Dictionary of the Medical Sciences]. 4th series, Vol. 5, p.403-584.

[Available on "Gallica", the website of digitized publications of the French national library at http://www.bnf.fr]

Bertillon (J.) 1879: Les célibataires, les veufs et les divorcés, au point de vue du mariage. [Inclination to marriage respectively of bachelors, widowers and divorced persons.] Revue Scientifique de la France et de l'Etranger 8,33,776-783.

Besnard (P.) 1997: Mariage et suicide. la théorie durkheimienne de la régulation conjugale à l'épreuve d'un siècle. [The impact of marriage on suicide revisited one century after Durkheim's work]. Revue Française de Sociologie, 38,735758.

Bojanovsky (J.) 1979: Wann droht der Selbstmord bei Geschiedenen? [At what point after separation are divorced people most likely to commit suicide?] Schweizer Archiv für Neurologie, Neurochirurgie und Psychiatrie 125,1,73-78.

Bojanovsky (J.) 1980: Wann droht der Selbstmord bei Verwitweten? [After becoming a widower when is the likelihood of committing suicide largest?]. Schweizer Archiv für Neurologie, Neurochirurgie und Psychiatrie 127,1,99-103.

Daguet (F.) 1995: Un siècle de démographie française. Structure et évolution de la population de 1901 à 1993. [Structure and evolution of the French population from 1901 to 1993], Institut National de la Statistique et des Etudes Economiques (INSEE), No 434-435, Paris.

Deaths: final data. National Vital Statistics Reports, Years 1996-2010. National Center for Health Statistics, Hyattsville, Maryland.

[available on the website of the NCHS at:

http://www.cdc.gov/nchs/products/nvsr.htm]

Durkheim (E.) 1897: Le suicide. Etude de sociologie. F. Alcan, Paris. A recent English translation is: "On Suicide" (2006), Penguin Books, London.

[Durkheim showed not only that suicide rates among non-married, widowed or divorced persons were higher than among married persons but also that they were lower among married persons with several children than for married persons without children.]

Farr (W.) 1859, 1975: Influence of marriage on the mortality of the French people (12 p.). Transactions of the National Association for the Promotion of Social Science 1858-1859, 504-520.

The paper was republished in 1975 in "Vital statistics, a memorial volume of selections from reports and writings of William Farr". Scarecrow Press, Methuen 
(New York). [The style of the report is somewhat outdated and sometimes confusing. An illustration is provided by the following excerpt taken from the first paragraph. "The action of the various parts of the body in industrial occupations produces specific effects. Every science modifies its cultivators. The play of the passions transfigures the human frame. How do they influence its existence?']

Flounders (S.) 2009: Why U.S. occupation cannot transform Afghanistan or Iraq. [available on the website of the "International Action Center"]

Frisch (M.), Simonsen (J.) 2013: Marriage, cohabitation and mortality in Denmark: national cohort study of 6.5 million persons followed for up to 3 decades. International Journal of Epidemiology 1,13.

Gompertz (B.) 1825: On the nature of the function expressive of the law of human mortality, and on a new mode of determining the value of life contingencies. Philosophical Transactions of the Royal Society 115,513585.

Gove (W.R.) 1972: Sex, marital status, and suicide. Journal of Health and Social Behavior 13,204-213.

Grove (R.D.), Hetzel (A.M.) 1968: Vital statistics rates in the United States, 19401960. United States Printing Office, Washington DC.

Helsing (K.J.), Szklo (M.), Comstock (G.W.) 1981: Factors associated with mortality after widowhood. American Journal of Public Health 71,802-809.

[The paper is based on the comparison of a sample of 4,032 persons who became widowed between 1963 and 1974 and a sample of same size of married persons. For males, the ratio of death rates of widowed persons to that of married persons is 1.34. The paper is freely available on the Internet.]

Koposova (A.J.), Breault (K.D.), Singh (G.K.) 1995: White man suicide in the United States. A multivariate individual-level analysis. Social Forces, 74,1,315323.

Linder (F.E.), Grove (R.D.) 1947: Vital statistics rates in the United States, 19001940. United States Printing Office, Washington DC.

March (L.) 1912: Some researches concerning the factors of mortality. Journal of the Royal Statistical Society 75,505-538.

[The paper includes a comparative analysis of mortality rates in France, Prussia and Sweden.]

Mellström (D.), Nilsson (A.), Odén (A.), Rundgren (A.), Svanborg (A.) 1982: Mortality among the widowed in Sweden. Scandinavian Journal of Social Medicine $10,33-41$.

[The study followed 360,000 individuals aged between 50 and 90 who were widowed in Sweden at some point between 1968 and 1978. It showed a peak 
in the mortality risk during the first 3 months. For widowers the amplitude (with respect to married persons) was 1.48 while for widows there was a peak of smaller amplitude, namely 1.22.]

Mortality statistics: review of the Registrar General on deaths in England and Wales. Series DHI, Number 16. Her Majesty's Stationary Office, London.

National Center for Health Statistics (NCHS) 1970: Mortality from selected causes by marital status. Vital and Health Statistics, Series 20, number 8.

Parkes (C.M.), Benjamin (B.), Fitzerald (R.G.) 1969: Broken heart. A statistical study of increased mortality among widowers. British Medical Journal 1,740743. [This is the continuation of the Young et al. (1963) study. The same sample was observed over 4 more years i.e. a total of 9 years after the death of the spouse. Over these 4 years the death ratio widowed/married was comprised between 0.90 and 0.95 .]

Raff (M.C.) 1998: Cell suicide for beginners. Nature 396, 12 November, 119-122.

Registrar General 1971: Statistical Review of England and Wales. Part III. Office of Population Censuses and Surveys. London.

Roehner (B.M.) 2007: Driving forces in physical, biological and socio-economic phenomena. Cambridge University Press, Cambridge.

Roehner (B.M.) 2014: Incidence of the Bertillon and Gompertz effects on the outcome of clinical trials. Physica A, 414, p. 300-307.

Statistisches Jahrbuch für die Bundesrepublik Deutschland 1978: Stuttgart.

Stroebe (W.), Stroebe (M.S.) 1987: Bereavement and health. The psychological and physical consequences of partner loss. Cambridge University Press, Cambridge. [The book is mostly concerned with psychological and other qualitative aspects. Although short (p. 151-167), the review of the quantitative evidence about the mortality of widowed persons is quite useful. It can be noted that the book has a broad reference section which contains about 600 entries.]

Thierry (X.) 1999: Risques de mortalité et de surmortalité au cours des 10 premières années de veuvage. [Excess mortality during the first 10 years of widowhood.] Population 54,2,177-204.

Thierry (X.) 2000: Risques de mortalité et causes médicales des décès aux divers moments du veuvage. Gérontologie et Société 95,27-43.

Vallin (J.), Meslé (F.), Valkonen (T.) 2001: Tendances en matière de mortalité et mortalité différentielle. Editions du Conseil de l'Europe, Strasbourg.

An English version was published under the title "Trends in mortality and differential mortality".

Wang (L.), Xu (Y.), Di (Z.), Roehner (B.M.) 2013: How does group interaction and 
its severance affect life expectancy? arXiv preprint 1304.2935 (9 April 2013)

Young (M.), Benjamin (B.), Wallis (C.) 1963: Mortality of widowers. Lancet 2,254256.

[This is a longitudinal study. The authors followed over a period of 5 years a sample of 4,486 widowers more than 55 years old and whose wives had died in January 1957 . They found a death rate ratio widowed/married of 1.39 in the first six months after the death of the spouse and 1.04 for the remaining time. The problem is that these ratios are too low to account for the ratio of 1.6 given by UK vital statistics data for age groups. On average individuals spend 5 years in a 10-year age group. If their death rate is multiplied by $k_{1}$ over an interval of $m$ months, and by $k_{2}$ for the remaining months, then for the 5 years during which they remain in the age group the average rate will be multiplied by: $k=$ $\left.m \times k_{1}+(60-m) \times k_{2}\right) / 60$; for $m=6, k_{1}=1.39, k_{2}=1.04$ one gets: $k=1.08$.] 